\title{
Psycholinguistic Markers of Therapeutic Rupture Types
}

\author{
Justin Jacques ${ }^{1}$ (D) https://orcid.org/0000-0002-8574-5380 \\ Cass Dykeman ${ }^{1}$ (D) https://orcid.org/0000-0001-7708-1409 \\ ${ }^{1}$ Oregon State University
}

This preprint has been prepared for review with a peer-reviewed journal. Comments are welcomed and can be directed to the first author at jacquesj@oregonstate.edu

\begin{abstract}
This study was designed to further understand rupture events that counselors encounter during a counseling session that ultimately impact the quality of the therapeutic alliance. We employed a cross-sectional analysis of a linguistic corpus created from mock counseling transcripts embedded in a website administered by a peer-reviewed expert in the psychology field and three video recorded sessions of Carl Rodgers, Fritz Pearls, and Albert Ellis. The content of the corpuses was analyzed using Linguistic Inquiry and Word Count software. The results showed a significant difference between she/he words, or third-person singular pronouns, and certainty words when comparing withdrawal and mixed rupture corpuses with a confrontation rupture corpus. In addition, we found a significant differences between positive emotion words and discrepancy words when comparing a rupture-infused psychotherapy corpus to a general psychotherapy corpus. Several implications for counseling and research are provided in response to these findings.

Keywords: corpus linguistics, therapeutic alliance, alliance rupture, rupture event, LIWC
\end{abstract}

\section{Introduction}

When an individual has a significant injury or is diagnosed with a serious disease, they search for the most well-known and highly qualified healthcare professional using the latest technologies to ensure they get the best treatment. However, in the field of counseling, there are few ways to identify a counseling superstar. If counselors could easily determine who was a counseling prodigy, the way educators train counselors would change drastically, and counseling outcomes would improve substantially. New software provides a way through the veil of words that compose a counseling session transcript to catch a glimpse at quality. With the aid of computer programs, researchers may be able to assess the skill of a counselor by examining the linguistic and psychological processes underlying their conversations with clients. Furthermore, this methodology may allow researchers to identify top counselors. This includes clinicians who could detect the most important components of effective counseling-namely, ruptures in the therapeutic alliance. The present study explored the therapeutic alliance by examining the linguistic and psychological features of the three rupture marker types: confrontation, withdrawal, and mixed. The study also identifies when these three rupture types occur, their frequency, and what this means for the quality of the counselor's relationship to the client and ultimately the proficiency of the counselor. 
There were two primary goals for the present study. First, we attempted to target gaps in the extant literature on alliance ruptures that occur in counseling sessions by helping the reader understand an area of ambiguity (understanding the linguistic and underlying psychological processes of rupture types) and investigating additional research questions (Farooq, 2017). Corpus linguistics research can examine a variety of topics using an assortment of corpora. These examinations have included everything from profanity use as a marker of distinction to understanding when second language learners implement the nuances of social dimensions in speaking tasks (Gablasova et al., 2017; McEnery, 2004). Concerning counseling research, few studies have appeared regarding the linguistics of any aspect of therapeutic alliance. Second, this study contributes to the counseling profession by disrupting current practice (Tadajewski \& Hewer, 2011). Research has also found that counselors do not improve their effectiveness through practical cumulative experience over their career (Chow et al., 2015) or understand why specific counselors can create and sustain a stronger therapeutic alliance than can their colleagues. The findings of this research could enable counselors and counselor educators to gain insight into this key practice dynamic and develop strategies to improve their therapeutic bond and efficacy (Newhill et al., 2000). In sum, the present study may provide counselors with additional insight into the subtle nuances of the three rupture marker types (withdrawal, confrontation, and mixed) to assist the repair of therapeutic alliance ruptures.

In the review of the literature on therapeutic alliance ruptures, eight themes emerged: (a) definition of alliance ruptures, (b) features of withdrawal rupture type, (c) features of confrontation type, (d) features of mixed rupture type, (e) research on the linguistics of alliance, (f) linguistic processes and alliance ruptures, (g) psychological processes and alliance ruptures, and ( $h$ ) broad psycholinguistic processes and alliance ruptures. After these eight themes are reviewed, the research questions that guided this are stated.

The existing literature provides a range of definitions of a therapeutic alliance rupture varying in terms of severity continuum, frequency, and type. The more severe ruptures on the continuum consist of impasses, empathic failures, dysfluencies, disturbances, disruptions, deteriorations, vicious circles or cycles, and breakdowns (Muran \& Safran, 2016). Subtle ruptures include weakenings, miscoordinations, strains, misattunements, misalliances, breaches, and enactments. In terms of frequency, a counselor may experience a single rupture, a few ruptures, or numerous ruptures within a counseling session, as therapeutic alliance ruptures vary in their presentation. In addition to severity and frequency, alliance ruptures can be classified by rupture type.

Research has divided therapeutic alliance ruptures into three subtypes or marker types: withdrawal, confrontation, and mixed rupture markers (Safran \& Muran, 2016). Construct validity of this tripartite model of ruptures has been supported by two articles-Safran and Muran (2006, 2019), which clearly outline the definitions of the therapeutic alliance rupture types and posit limitations to these proposed constructs. Concerning this model, Safran et al. (2011) meta-analysis gave evidence that identifying and repairing ruptures were positively related to a beneficial therapeutic outcome. Given a review of the research supporting the construct validity of this tripartite, an examination of each part is warranted. 
Withdrawal rupture markers consist of behaviors that indicate a disconnection in the counseling relationship, evidence of the client moving away from the counselor, and a subtle alliance rupture event (Muran \& Safran, 2016). The client most commonly communicates their disconnect from the counselor by displaying behaviors that represent disengagement from either the counselor, some aspect of counseling, or an affective state. Salient examples include abstract talk, storytelling, minimal responses, topic shifts, and silences. Additionally, withdrawal ruptures create distance by moving the client away from the counselor and toward independence (Muran \& Safran, 2016). Examples include overt denial or a client's words not matching their affect.

Furthermore, withdrawal ruptures are subtle and consist of difficult-to-identify words and behaviors. Taking a self-critical stance in a counseling session or appeasing a counselor are examples. In general, withdrawal rupture markers are difficult to detect because the client's covert language and behavior represent a disconnection and distancing process.

In direct contrast to withdrawal ruptures are confrontation ruptures. Confrontation rupture markers are detectable breaks in the counseling relationship between the counselor and client and occur in an antagonistic manner. The primary characteristic of a confrontation rupture marker is the explicit communication of frustration or discontentment by the client to the counselor. In addition, these rupture types consist of movements against the counselor with an antagonistic posture and a bid for relational control. Confrontation ruptures may also include coercions such as being excessively friendly or even seductive (Eubanks et al., 2018). Salient examples include concerns or conflicts about: (a) the course of counseling, (b) the procedure of counseling, (c) the limits of counseling, (d) the activities of counseling, (e) a counselor in session activities, and (f) the counselor (Eubanks et al., 2015). Because of their direct nature, confrontation rupture markers are easier to identify and are more obvious to the counselor, but they still signify an injury to the counseling relationship.

A final rupture marker type, called a mixed rupture, is a combination of the confrontation and withdrawal rupture marker types. Mixed ruptures are composed of elements from both withdrawal and confrontation rupture markers. Within a mixed rupture, the client moves away and against the counselor concurrently, or in a sequence of verbal interactions. An example is when a client overtly disagrees with their counselor about an interpretation they have made, which represents a confrontation, but then immediately retracks their claim to reduce tension, in an act of withdrawal. This example highlights that clients often exhibit a combination of confrontation and withdrawal markers at times when they are upset about some aspect of counseling but at the same time want to avoid conflict with their counselor (Eubanks et al., 2015). Mixed rupture markers have the most complex presentation of the three rupture types and may be the most difficult to identify. Hence, it may be beneficial to utilize instruments that can assist counselors in identifying the various rupture marker types effectively and efficiently.

A common way to identify the various rupture markers is through the analysis of counseling session transcriptions. Recently, linguistic researchers have utilized software programs to code and subsequently evaluate counseling sessions (Perez-Rosas et al., 
2017). These automated programs can reliably and efficiently code and summarize large amounts of transcription-based data in a matter of minutes. These tools assist linguists in their research endeavors and counselors in their session transcription coding tasks (Perez-Rosas et al., 2017). Two noteworthy software programs that can perform counseling session transcription analysis are Language Inquiry and Word Count (LIWC; Pennebaker et al., 2015c) and Antconc (Anthony, 2017). These software platforms can uncover and code underlying counseling and client linguistic processes through the analysis of gram patterns, which are parallel linguistic patterns between counselors' and clients' speech, as well as context-dependent metafeatures. Metafeatures are random framework-like elements that exist behind language. They are a collection of more common aspects of language that are identified by grouping trait occurrences from automatically annotated transcription data. Metafeatures may enable researchers to uncover significant relationships amongst words within a set of interactions (Chen et al., 2016) in this instance, a counseling session. As metafeatures are discovered and their covert significances understood, they can be employed to analyze counseling session conversations and subsequently assess the strength of the alliance between the counselor and client. Additionally, associated metafeatures could help researchers identify dialog cycles between client and counselor. Linguistic-oriented computer programs such as LIWC and Antconc are linguistic devices that can help social scientists more precisely evaluate gram patterns and their hidden metafeatures, which are numerous in the semantic dialogues that make up the therapeutic alliance.

There exist several linguistic variables that may presage alliance rupture. A salient example of a linguistic variable that can be measured is pronoun use. Simmons et al. (20005) found that when couples were asked to rate their marriages for an interviewer, the more they used "we," the better their marriage. Additionally, research has shown that using the pronoun "you" in conversation predicts lower relationship quality (Tausczik \& Pennebaker, 2010). Other studies have found that second-person pronoun use (e.g., you, your) was negatively related to the quality of a relationship (Simmons et al., 2008). Because therapeutic alliance is analogous to a marriage or a close relationship, assessing pronoun use could indicate the quality of the alliance and help target alliance ruptures. Emotional language use is another linguistic variable helpful in identifying alliance ruptures. In written discourse, negative emotion words (e.g., hurt, ugly, nasty) are used in writing about negative events (Kahn et al., 2007). Analyzing negative emotion words in verbal discourse may signal negative events and reveal ruptures in the therapeutic alliance, specifically when the counselor and client are talking about their counseling relationship.

An additional linguistic variable that can help identify a therapeutic rupture is word count. Word count analysis can illuminate who is controlling the conversation and how engaged the parties are in the discourse (Tausczik \& Pennebaker, 2010). In a study of social hierarchy, Sexton and Helmreich (2000) found that individuals of high status tend to speak more frequently and openly and make more statements that involve others. Conversely, low-status speakers have the propensity to use language that is more self-focused and cautious. In the therapeutic relationship, where status tends to be more equal and the client typically speaks more often than the counselor, identifying unequal status and power dynamics through discourse analysis could help identify alliance rupture. Word count is also helpful in assessing level of engagement (Tausczik \& 
Pennebaker, 2010) and may help identify when a client is subtly or overtly disengaged from a counseling session signifying a rupture. Finally, the number and frequency of acquiesces and positive emotion words are helpful in measuring levels of agreement (Tausczik \& Pennebaker, 2010). Assessing the level of agreement in a counseling session could, in turn, point to a positive alliance or detect a subtle rupture, depending on the context.

There are numerous psychological processes that signify alliance events and outcomes. Pennebaker et al. (2015b) included the following variables as categories in their linguistic-based measure of psychological processes: time orientation, positive emotion, negative emotion, anxiety, anger, and sadness. A foundational study conducted by Mergenthaler and Bucci (1999) discovered that by analyzing three categories of words-emotional tone, abstraction, and referential activity-they could predict successful outcomes in therapy. To discern emotional tone, Mergenthaler and Bucci tracked the flow of the therapeutic discourse, targeted the patients' verbal form, and looked for expressions of emotional experience. Another study looking at the negative emotion variable embedded in a psychological process measure found that better therapy outcomes were associated with a reduction in negative emotion words over the course of treatment when working with clients diagnosed with a personality disorder (Arntz et al., 2012). These studies support the use of linguistic methodologies to identify psychological processes that presage.

Given the aforementioned, three research questions were developed to guide this study. These questions were:

RQ1: What is the use rate of linguistic and psychological processes of ruptureinfused psychotherapy by type?

RQ2: Do linguistic and psychological processes differ by rupture type in ruptureinfused psychotherapy? If so, how do they differ?

RQ3: Does the use rate of linguistic and psychological processes in ruptureinfused psychotherapy type differ from the use rate of these processes in general psychotherapy?

\section{Design}

\section{Method}

This study utilized a synchronic corpus linguistic design (Brezina, 2018). The corpus was created by transcribing mock counseling vignettes obtained from the "clinical tools" subheading on a publicly available website created by nationally recognized experts in alliance ruptures and three video recorded sessions of Carl Rodgers', Fritz Pearls', and Albert Ellis' work from the Three Approaches to Psychotherapy films (Shostrom, 1965). The study involved 14 variables. The four linguistic process variables examined were: first-person singular pronouns, first-person plural pronouns, third-person singular pronouns, and third-person plural pronouns. The 10 psychological process variables explored were: negative emotion, anger, sadness, anxiety, positive emotion, discrepancy, certainty, differentiation, tentative, and causation. The unit of analysis was single words (Bjekić et al., 2014). 
An a priori power analysis $\chi 2$ test square test was conducted using $G *$ Power 3.1 (Faul et al., 2009). The proper effect size for such a test was Cohen's w (Rosnow \& Rosenthal, 2003). The effect size input for this power analysis was drawn from an online environment study of gender discourse (Sullivan et al., 2015). The input parameters were: (a) test family - $\chi 2$ tests; (b) statistical test - goodness-of-fit tests: contingency tables; (c) type of power analysis - a priori: compute required sample size - given $\alpha$, power, and effect size; (d) $w=0.60$; (e) power (1- $\beta$ error probability) $=0.90$; (f) $\alpha=$ .0001 ; and $(\mathrm{g})$ degrees of freedom (Df) $=2$. The $\mathrm{G}^{*}$ Power 3.1 output suggested a sample size of 67 with an actual power of 0.91 .

\section{Corpus}

\section{Register, Scope, and Sources}

Biber (2012) detailed the existence of four main registers in English: (a) conversation, (b) fiction, (c) news reportage, and (d) academic prose. The texts of the present study fall within the conversation register with the subregister being psychotherapy conversation. In building the corpus, researchers used transcriptions from mock counseling vignette videos that highlighted the three types of alliance ruptures (confrontation, withdrawal, and mixed) based on the constructs from Muran and Safran's seminal psychotherapy research (Muran \& Safran, 2016). The videos were obtained from their website (https://www.therapeutic-alliance.org/) under the "clinical tools" tab (Muran et al., n.d.). Dr. Muran, who is a nationally renowned expert in ruptures, described the process of making the videos for the website:

For the videos on our website, we simply asked our students to bring and play difficult moments (some based on their readings, some based on their own clinical experience). We specifically invited withdrawal, confrontation and mixed rupture events and kept it to one take to promote authenticity and spontaneity: Only the initial rupture marker and case formulation were discussed in advance. The students were familiar with our definitions and principles. (C. J. Muran, personal communication, June 18, 2021)

These publicly available mock counseling sessions were transcribed for the creation of the corpus. This included the transcription of three counseling vignettes that highlighted confrontation ruptures, two vignettes that highlighted withdrawal ruptures, and four vignettes that highlighted mixed ruptures. The resultant confrontation rupture corpus contained three transcripts, 1,995 tokens, and 387 types; the resultant withdrawal rupture corpus contained two transcripts, 2,384 tokens, and 393 types; and the resultant mixed rupture corpus contained four transcripts, 3,148 tokens, and 513 types.

Additionally, a reference corpus was created to establish a base rate for psychological processes within a therapy session. The corpus was created by combining the transcriptions of the emblematic and influential counseling sessions with Carl 
Rogers, Fritz Pearls, and Albert Ellis (Shostrom, 1965). The resultant reference corpus contained three transcripts, 13,584 tokens, and 1,410 types.

\section{Preprocessing}

Nine mock counseling vignette videos (three confrontation, two withdrawal, and four mixed) were disembedded from the therapeutic-alliance.org website (Muran et al., n.d.) using standard downloading protocol and were subsequently converted into MP4 files. The files were then uploaded to Transcribe.wreally.com and electronically transcribed. Transcripts were subsequently converted into Word documents after being manually checked for transcription accuracy. Additionally, three video recorded sessions of Carl Rodgers, Fritz Pearls, and Albert Ellis working with clients were transcribed from the Three Approaches to Psychotherapy films (Shostrom, 1965). The electronic files were then converted into .txt files using AntFileConverter (Anthony, 2017), and three distinct corpuses were created by combining the respective .txt files of each rupture type vignette videos (confrontation, withdrawal, or mixed). Spelling and word-related errors were identified and corrected, and the corpora were further cleaned for nonASCII characters and diacritics using After the three rupture type corpuses were preprocessed, they contained 1,995 (confrontation), 2,384 (withdrawal), 3,148 (mixed), and 13,584 (Carl Rodgers, Fritz Pearls, and Albert Ellis) words.

\section{Measures}

Linguistic Inquiry and Word Count (LIWC; Pennebaker et al., 2015c) was the software program used in this analysis. Within LIWC, there are multiple subscale measures that can be utilized to analyze a corpus. The validity and reliability of LIWC have been well established (Pennebaker et al., 2015a).

\section{Linguistics Processes}

The LIWC linguistics processes employed were first-person singular ("I, me, my, mine"), first-person plural ( "we," "us," "our," and "ours"), third-person singular ("she," "her," and "him"), and third-person plural pronouns ("they," "their," and "they' $d$ ") (Pennebaker et al., 2015b).

\section{Psychological Processes}

The LIWC psychological processes employed were: negative emotion (e.g., "hurt"), anger (e.g., "hate"), sadness, (e.g., "lonely"), anxiety (e.g., "worried"), positive emotion (e.g., "happy"), discrepancy (e.g., "should"), certainty (e.g., "always"), differentiation (e.g., "hasn't"), tentative (e.g., "maybe"), and causation (e.g., "because"; Pennebaker et al., 2015b).

\section{Apparatus}

Linguistic Inquiry Word Count (LIWC) 
The most recent edition of the LIWC software is from 2015 (Pennebaker et al., 2015c). The default LIWC2015 dictionary is composed of almost 6,400 words, word stems, and sect emoticons. The program is also composed of 90 analyzable output variables, which focus mainly on psychological processes. The variables are scored by comparing the percentage of words being analyzed to a dictionary of words in categories and subdictionaries (Smith-Keiling \& Hyun, 2019).

\section{Data Analysis}

For RQ1 (use rates), raw and normalized frequency rates (i.e., percentage of all words) are reported for all variables across all three types of alliance ruptures (confrontation, withdrawal, and mixed). Normalized frequencies (e.g., relative frequencies) were calculated by taking the percentage of the total words of a variable (e.g., she/he words were .0015 percent of confrontation corpus) as calculated by the LIWC software and dividing that number by 100 (basis for normalization) and then multiplying the result by the total number of tokens (Brezina, 2018). In terms of RQ2, (type differences), the log likelihood test (G2) was employed (Rayson \& Garside, 2000). The effect size was calculated using the Bayes information criterion (BIC) with interpretation guidelines from Wilson (2013). If overall significant type differences were encountered, post hoc pairwise comparisons were conducted using the $\mathrm{G} 2$ test (McDonald, 2014). Concerning RQ3, differences between the rupture and general counseling corpuses were assessed by means of $\mathrm{G} 2$ and BIC. All analyses were conducted using $\mathrm{R}$ with a preset alpha level of .001. Additionally, due to the large number of tests, a Bonferroni correction was utilized to set a family-wise error rate to control for type one errors.

\section{Results}

Regarding RQ1, the raw and normalized count of linguistic and psychological processes for confrontation, withdrawal, and mixed rupture type corpuses can be found in Table 1.1. In terms of RQ2, differences in the linguistic and psychological processes category among the rupture types can be reviewed in Table 1.1 For those linguistic and psychological processes where significant differences did occur, the pairwise post hoc analyses can be inspected in Table 1.2. Concerning RQ3, linguistic and psychological processes that distinguished the rupture corpus from the baseline counseling corpus can be examined in Table 1.3.

\section{Discussion}

The aim of this study was to explore the linguistic and psychological processes that take place in mock counseling session vignettes that consisted of an alliance rupture event. In this section the potential reasons for the obtained results are

discussed. After this discussion, limitations, practice implications, and researched implications are addressed. RQ1 looked at the level of use of linguistic and psychological processes known to be related to each of the respective alliance rupture types 
(confrontation, withdrawal, and mixed). The probable explanations for these obtained results are addressed in the discussions in the findings for RQ2 and RQ3.

Regarding RQ2, two of the 14 variables returned a significant result. The first of these was the third-person singular pronoun variable. Two likely explanations for this result should be considered. First, research has found that third-person pronouns have been associated with self-monitoring and general social awareness in verbal discourse (Pennebaker et al., 2003). This could be one explanation as to why she/he words were found to be significantly more common in withdrawal and mixed ruptures compared to confrontation ruptures, as a client or counselor may be more subtlety aware of themselves and their relationship with the other in these events. A second explanation is that pronoun use also indicates the focus of the speaker's attention (Kacewicz et al., 2014) and in the case of third-person singular pronouns, a focus on others. This aligns with withdrawal rupture events and mixed events (which include withdrawal rupture events), where the client is subtly moving away from the counselor (Muran \& Safran, 2016) and may include an "other focus" by the client to hide the misattunement. Both findings seem plausible, but the first finding appears more likely as confrontation ruptures may be other focused but are, by definition, disagreements.

The second significant result was for the certain variable. Two likely explanations for this result should be considered. The first explanation is that "certain words" may represent the speaker's perceived sense of power from a psycholinguistic perspective. Research has shown that those who use certainty language (e.g., always, never) in verbal discourse are viewed as more powerful (Adkins \& Brashers, 1995; Han \& Lind, 2017; Hart \& Childers, 2004) and are more committed to the truth of what they are asserting (Holmes, 1982). This finding makes logical sense in the context of a confrontation rupture where a client is directly expressing anger or dissatisfaction toward the counselor (Muran \& Safran, 2016). This subsequent certainty language employed by the client or counselor may reflect their commitment to their perceived truth and be seen by the observer (counselor or client) as an act of power. A secondary explanation is that research shows they increase the persuasiveness of a message (Corley \& Wedeking, 2014). This makes logical sense in that a confrontation rupture is where a client is moving toward the counselor in a direct manner (Eubanks et al., 2016) or bringing up a concern or complaint regarding the counselor or some aspect of counseling in a targeted manner (Muran \& Safran, 2016). According to Corley and Wedeking, certainty language is often used to ensure compliance. Within a confrontation rupture event, the client may want the counselor to comply with their request after directly confronting them about their personal concerns about the counselor or some aspect of the counseling. Both findings may be plausible, but the second finding appears more likely as clients often want the counselor to comply with their requests regarding some goal or task related to counseling process. However, because the $\mathrm{BIC}$ was under 2, at 1.52, caution should be taken in drawing strong conclusions unless this finding can be replicated or other evidence is presented, as the results are weak and may have occurred by chance.

In the matter of RQ3, two explanations deserve consideration for each finding. First, pertaining the finding of a decreased use of positive emotion words for ruptureinfused psychotherapy (general psychotherapy comparison), the use of positive emotion 
words has been shown to help stabilize emotionality because an individual is able to shift attention away from self (Lyons et al., 2006). This would also make logical sense in the context of an alliance rupture event where both the client and counselor may be using less positive emotion words as there is disharmony in either of the party's internal states, as overt or covert conflict is occurring in the counseling session. Second, positive emotion words have been shown to correlate to social coping. This finding makes sense in the context of a rupture event (confrontation, withdrawal, or mixed), as the client and even the counselor may not be using social coping (Chung \& Pennebaker, 2012) to sustain social norms. Both findings are plausible, but the second finding is more convincing as social coping to sustain social norms is necessary in communication and appears very important in the context of an alliance rupture event.

Regarding the second finding, there are two plausible explanations for the obtained result of significantly lower use of "discrepancy words." First, this possibly counterintuitive finding (the reader might expect that rupture events in counseling discourse would be more cognitive in nature) could be explained by the fact that therapy is inherently composed of cognitive mechanisms, or language which includes discrepancy words (Lee et al., 2011). This includes counselors and clients discussing their thoughts (cognitions) about causes, consequences, or conflict about a discussion topic (Chung \& Penebaker, 2012). Therefore, both the rupture infused corpus and the general psychotherapy corpus may have higher than usual cognitive content or discrepancy language than other verbal discourse. Second, the lower number of discrepancy words, a subset of cognitive processing words, in the rupture infused corpus compared to the general psychotherapy corpus could be explained by an unproductive moment in therapy. Ruptures have been defined as missattunements between the client and counselor (Muran \& Safran, 2016), and in those segments of therapy discourse the conversation may be less cognitive in nature, on average, than in baseline or useful therapy where the counselor and client are attuned. This explanation aligns with research showing that highly helpful or productive moments in therapy include greater proportion of cognitive words and specifically words indicating insight (McCarthy et al., 2017).

Additionally, an increased frequency of discrepancy words such as "should" or "would" has been shown to be accompanied by an overall decrease or weakening in clout (certainty) and confidence in written text (Moore et al., 2021). The findings by Moore et al. also align with the statistically significant finding of discrepancy words, which shows lower certainty (clout) and less confidence by the client or counselor. In the context of rupture events, clients may be less certain and confident about the counseling process and may communicate this through subtle passive communication in the case of a withdrawal rupture. Or, they may display a lack of certainty or confidence in a direct confrontation with the counselor in the case of a confrontation rupture event. Equally important, the counselor may reciprocate in one of the three rupture events with a plethora of discrepancy words, consciously or unconsciously communicating their own decrease in certainty or confidence through verbal discourse. Both justifications seem to have merit, but the first explanation is more plausible, as the cognitive nature of psychotherapy conversations around conflict, both active and passive, is very apparent. 
Two limitations to the present study should be noted. The first limitation is sample size. Having a larger sample would allow for a more in-depth exploration of the vocabulary related to each of the rupture types and allow for additional experiments on a larger number of areas. In order to identify the underlying linguistic and psychological processes that occur in a rupture event, studies would require a much larger corpus and one that would separate the clients' and counselors' discourses. Additionally, there are some significant limits regarding our capacity to generalize the findings from this study to other alliance rupture discourse datasets. First, we utilized a corpus of a mock counseling session, which included professional counselors and actors. Although this set of discourses are very similar to an actual counseling session, there are likely significant differences between the discourse of a session that included playacting with a counselor and an actual counseling session discourse. A second limitation is that analyses focus on whole sessions rather than specific rupture events. Results may have varied if the discourse around each rupture event had been isolated for a study.

There are four implications for counseling practice that can be drawn from the obtained results. First, the finding of she/he words that indicates the necessity of supervisors and counselors to remain keenly aware of increased use of third-person singular pronouns by their clients (and their own use) is important. This is because third person singular pronoun use may indicate self-monitoring and increased general social awareness in verbal discourse (Pennebaker et al., 2003) and may indicate a withdrawal or mixed rupture event. Furthermore, supervisors and counselors should be aware that pronoun use point to the focus of the speaker's attention and specifically third-person singular pronoun use (she/he words) is "other focused" (Kacewicz et al., 2014). This may signal that a client in the context of a withdrawal or mixed rupture may be using deflection of attention to create distance in a subtle manner as they are covertly moving away from the counselor or some aspect of the counseling process.

Second, the finding of certainty words necessitates that supervisors and counselors remain keenly aware of increased certainty language in counseling session discourse. This finding is important because it may indicate that the client or the counselor believes the other is in a position of power, are more committed to certainty (Adkins \& Brashers, 1995; Han \& Lind, 2017; Hart \& Childers, 2004; Holmes, 1982), and that a rupture event is occurring. Additionally, because there is evidence that using certainty language increases the persuasiveness of their message, supervisors and counselors need be aware of this type of discourse (in the context of a confrontation rupture event) to call out and work through a potential rupture in the therapeutic alliance with a client.

Third, the finding of infrequent use of positive emotion words in the rupture infused psychotherapy corpora indicates the necessity of supervisors and counselors to remain keenly aware of a decrease of positive emotion language in counseling session discourse. This is important because it may indicate that a participant is feeling less emotionally stable (Lyons et al., 2006), may not be coping socially, and be less able to sustain the social norms of conversation (Chung \& Pennebaker, 2012). Therefore, a decrease in positive emotion words may signify a subtle disconnect in the therapeutic alliance, which counselors could become skilled at recognizing and repairing. 
A final implication regarding the finding of decreased use of discrepancy words in the rupture infused psychotherapy corpus is that this may be an indicator that the discourse may be less cognitive in nature than the average psychotherapy conversation. This lack of discrepancy words, which are cognitive in nature, may indicate that a rupture event is occurring, and that the session or segment is not as therapeutically useful (McCarthy et al., 2017) because the counselor and client are not attuned, and helpful cognitive insights are not occurring. Supervisors and counselors who recognize a decrease in discrepancy or cognitive language can be aware that a rupture event may have occurred, attempt to disembed from the event, and subsequently tend to the discord in the counseling relationship.

Two recommendations for further research should be noted. First, because the results demonstrate that the linguistic and psychological process that underlies language between three types of alliance rupture types may be distinguishable, researchers have a new opportunity to further explore rupture-specific counseling session discourse. Specifically, it may be important for researchers to continue this line of inquiry and study each rupture type (confrontation, withdrawal, and mixed) and their discourse in actual counseling sessions. Second, expanding on the results of this study would allow researchers to further examine alliance ruptures but in a more granular manner through analyzing rupture marker events in counseling session segments. 


\section{References}

Adkins, M., \& Brashers, D. E. (1995). The power of language in computer-mediated groups. Management Communication Quarterly, 8(3), 289-322. https://doi.org/10.1177\%2F0893318995008003002

Althoff, T., Clark, K., \& Leskovec, J. (2016). Large-scale analysis of counseling conversations: An application of natural language processing to mental health. Transactions of the Association for Computational Linguistics, 4, 463-476. https://doi.org/10.1162/tacl a 00111

Anthony, L. (2017). AntFileConverter (Version 1.2. 1) [software]. https://www.laurenceanthony.net/software/antfileconverter/

Arntz, A., Hawke, L. D., Bamelis, L., Spinhoven, P., \& Molendijk, M. L. (2012). Changes in natural language use as an indicator of psychotherapeutic change in personality disorders. Behaviour Research and Therapy, 50(3), 191-202. https://eprints.lancs.ac.uk/id/eprint/51045

Biber, D. E. (2012). Corpus-based and corpus-driven analyses of language variation and use. In B. Heine \& H. Narrog (Eds.), The Oxford handbook of linguistic analysis (pp. 193-224). Oxford University Press.

Bjekić, J., Lazarević, L. B., Živanović, M., \& Knežević, G. (2014). Psychometric evaluation of the Serbian dictionary for automatic text analysis-LIWCser. Psihologija, 47(1), 5-32.

https://doi.org/10.2298/PSI1401005B

Brezina, V. (2018). Statistics in corpus linguistics: A practical guide. Cambridge University Press. Chen, J., Qiu, L., \& Ho, M. H. R. (2020). A meta-analysis of linguistic markers of extraversion: Positive emotion and social process words. Journal of Research in Personality, 89, Article 104035. https://doi.org/10.1016/i.jrp.2020.104035

Chen, W., Zhang, M., Zhang, Y., \& Duan, X. (2016). Exploiting meta features for dependency parsing and part-of-speech tagging. Artificial Intelligence, 230(C), 173-191.

https://doi.org/10.1016/i.artint.2015.09.002

Chow, D. L., Miller, S. D., Seidel, J. A., Kane, R. T., Thornton, J. A., \& Andrews, W. P. (2015). The role of deliberate practice in the development of highly effective psychotherapists. Psychotherapy, 52(3), 337345. https://doi.org/10.1037/pst0000015

Chung, C. K., \& Pennebaker, J. W. (2012). Linguistic inquiry and word count (LIWC): pronounced "Luke,"... and other useful facts. In Applied natural language processing: Identification, investigation and resolution (pp. 206-229). IGI Global.

Cohn, M. A., Mehl, M. R., \& Pennebaker, J. W. (2004). Linguistic markers of psychological change surrounding September 11, 2001. Psychological Science, 15(10), 687-693.

https://doi.org/10.1111\%2Fj.0956-7976.2004.00741.x 
Corley, P. C., \& Wedeking, J. (2014). The (dis) advantage of certainty: The importance of certainty in language. Law \& Society Review, 48(1), 35-62. https://doi.org/10.1111/lasr.12058

Decter-Frain, A., \& Frimer, J. A. (2016). Impressive words: Linguistic predictors of public approval of the US Congress. Frontiers in Psychology, 7, Article 240. https://doi.org/10.3389/fpsyg.2016.00240

Ellington, L., Kelly, K. M., Reblin, M., Latimer, S., \& Roter, D. (2011). Communication in genetic counseling: Cognitive and emotional processing. Health Communication, 26(7), 667-675. https://doi.org/10.1080/10410236.2011.561921

Eubanks, C. F., Lubitz, J., Muran, J. C., \& Safran, J. D. (2019). Rupture resolution rating system (3RS): Development and validation. Psychotherapy Research, 29(3), 306-319. https://doi.org/10.1080/10503307.2018.155203 4

Eubanks, C. F., Muran, J. C., \& Safran, J. D. (2018). Alliance rupture repair: A meta-analysis. Psychotherapy, 55(4), 508-519. https://doi.org/10.1037/pst0000185

Farooq, R. (2017). A framework for identifying research gap in social sciences: Evidence from the past. IUP Journal of Management Research, 16(4), 66-75. https://www.iupindia.in/705/ijmr.asp Fast, E., \& Horvitz, E. (2016). Identifying dogmatism in social media: Signals and models. https://arxiv.org/abs/1609.00425v1

Faul, F., Erdfelder, E., Buchner, A., \& Lang, A. G. (2009). Statistical power analysis using G*Power 3.1: Test for correlation and regression analysis. Behavioral Research Methods, 41(4), 1149-1160. https://doi.org.10.3758/BRM.41.4.1149

Gablasova, D., Brezina, V., McEnery, T., \& Boyd, E. (2017). Epistemic stance in spoken L2 English: The effect of task and speaker style. Applied Linguistics, 38(5), 613-637.

https://doi.org/10.1093/applin/amv055

Gill, A. J., French, R. M., Gergle, D., \& Oberlander, J. (2008, November). The language of emotion in short blog texts. In Proceedings of the 2008 ACM conference on Computer supported cooperative work (pp. 299-302). Association for Computing Machinery. https://doi.org/10.1145/1460563.1460612

Gunsch, M. A., Brownlow, S., Haynes, S. E., \& Mabe, Z. (2000). Differential forms linguistic content of various of political advertising. Journal of Broadcasting \& Electronic Media, 44(1), 27-42.

https://doi.org/10.1207/s15506878jobem4401 3

Han, S. H., \& Lind, C. J. (2017). Putting powerfulness in its place: a study on discursive style in public discussion and its impact. Argumentation and Advocacy, 53(3), 216-233.

https://doi.org/10.1080/00028533.2017.1337332

Hart, R. P., \& Childers, J. P. (2004). Verbal certainty in American politics: An overview and extension. Presidential Studies Quarterly, 34(3), 516-535. https://doi.org/10.1080/00028533.2017.1337332 
Hartung, F., Burke, M., Hagoort, P., \& Willems, R. M. (2016). Taking perspective: Personal pronouns affect experiential aspects of literary reading. PloS One, 11(5).

https://doi.org/10.1371/journal.pone.015473 2

Holmes, J. (1982). Expressing doubt and certainty in English. RELC journal, 13(2), 9-28.

https://doi.org/10.1017/S0047404500011623

Huang, C.-L., Chung, C. K., Hui, N., Lin, Y.-C., Seih, Y.-T., Lam, B. C. P., Chen, W.-C., Bond, M. H., \& Pennebaker, J. W. (2012). The development of the Chinese linguistic inquiry and word count dictionary. Chinese Journal of Psychology, 54(2), 185-201. https://psycnet.apa.org/record/2014-20116-004

Kacewicz, E., Pennebaker, J. W., Davis, M., Jeon, M., \& Graesser, A. C. (2014). Pronoun use reflects standings in social hierarchies. Journal of Language and Social Psychology, 33(2), 125-143. https://doi.org/10.1177\%2F0261927X13502654

Kahn, J. H., Tobin, R. M., Massey, A. E., \& Anderson, J. A. (2007). Measuring emotional expression with the Linguistic Inquiry and Word Count. American Journal of Psychology, 120(2), 263-286. https://doi.org/10.2307/20445398

Liu, D., \& Lei, L. (2018). The appeal to political sentiment: An analysis of Donald Trump's and Hillary Clinton's speech themes and discourse strategies in the 2016 US presidential election. Discourse, Context \& Media, 25, 143-152. https://doi.org/10.1016/j.dcm.2018.05.001

Lyons, E. J., Mehl, M. R., \& Pennebaker, J. W. (2006). Pro-anorexics and recovering anorexics differ in their linguistic Internet self-presentation. Journal of Psychosomatic Research, 60(3), 253-256. https://doi:10.1016/i.jpsychores.2005.07.017

McCarthy, K. L., Caputi, P., \& Grenyer, B. F. (2017). Significant change events in psychodynamic psychotherapy: Is cognition or emotion more important?. Psychology and Psychotherapy: Theory, Research and Practice, 90(3), 377-388. https://doi.org/10.1111/papt.12116

McDonald, R. P. (2014). Factor analysis and related methods. Psychology Press.

McEnery, T. (2004). Swearing in English: Bad language, purity and power from 1586 to the present. Routledge.

Moons, W. G., Spoor, J. R., Kalomiris, A. E., \& Rizk, M. K. (2013). Certainty broadcasts risk preferences: Verbal and nonverbal cues to risk-taking. Journal of Nonverbal Behavior, 37(2), 79-89. https://doi.org/10.1007/s10919-013-0146-0

Muran, J. C., Eubanks, C. F. \& Samstag L.W. (n.d.). Clinical tools. https://www.therapeuticalliance.org/clinical-tools.html 
Muran, J. C., \& Safran, J. D. (2016). Therapeutic alliance ruptures. In A.Wenzel (Ed.), Sage encyclopedia of abnormal \& clinical psychology (pp. 3,512-3,514). Sage Publications.

Newman, M. L., Pennebaker, J. W., Berry, D. S., \& Richards, J. M. (2003). Lying words: Predicting deception from linguistic styles. Personality and Social Psychology Bulletin, 29(5), 665-675.

https://doi.org/10.1177\%2F0146167203029005010

Pennebaker, J. W. (1997). Writing about emotional experiences as a therapeutic process. Psychological Science, 8(3), 162-166. https://doi.org/10.1111\%2Fj.1467-9280.1997.tb00403.x

Pennebaker, J. W. (2011). The secret life of pronouns. New Scientist, 211(2828), 42-45.

https://doi.org/10.1016/S0262-4079(11)62167-2

Pennebaker, J. W., Booth, R. J., Boyd, R. L., \& Francis, M. E. (2015a). LIWC 2015 operator's manual. Pennebaker Conglomerates.

Pennebaker, J. W., Boyd, R. L., Jordan, K., \& Blackburn, K. (2015b). The development and psychometric properties of LIWC2015. http://hdl.handle.net/2152/31333

Pennebaker, J. W., Francis, M. E., \& Booth, R. J. (2015c). Linguistic Inquiry and Word Count: LIWC2015. (Version 1.6) [Computer software]. Pennebaker Conglomerates. https://liwc.wpengine.com

Pennebaker, J. W., Mehl, M. R., \& Niederhoffer, K. G. (2003). Psychological aspects of natural language use: Our words, our selves. Annual Review of Psychology, 54(1), 547-577.

https://doi.org/10.1146/annurev.psych.54.101601.145041

Pérez-Rosas, V., Mihalcea, R., Resnicow, K., Singh, S., An, L., Goggin, K. J., \& Catley, D. (2017, April). Predicting counselor behaviors in motivational interviewing encounters. In Proceedings of the 15th Conference of the European Chapter of the Association for Computational Linguistics: Volume 1, Long Papers (pp. 1,128-1,137). Association for Computational Linguistics.

https://www.aclweb.org/anthology/E17-1106.pdf

Rayson, P., \& Garside, R. (2000, October). Comparing corpora using frequency profiling. In Proceedings of the workshop on comparing corpora-Volume 9 (pp. 1-6). Association for Computational Linguistics. https://doi-org.ezproxy.proxy.library.oregonstate.edu/10.3115/1117729.1117730

Rosnow, R. L., \& Rosenthal, R. (2003). Effect sizes for experimenting psychologists. Canadian Journal of Experimental Psychology/Revue Canadienne de Pexpérimentale, 57(3), 221-237. https://psycnet.apa.org/doi/10.1037/h0087427

Sexton, J. B., \& Helmreich, R. L. (2000). Analyzing cockpit communications: The links between language, performance, and workload. Human Performance in Extreme Environments, 5(1), 63-68.

https://doi.org/10.7771/2327-2937.1007 
Shostrom, E. L. (Director). (1965). Three approaches to psychotherapy [Film]. Santa Ana, CA: Psychological Films.

Simmons, R. A., Gordon, P. C., \& Chambless, D. L. (2005). Pronouns in marital interaction: What do "you" and "I" say about marital health? Psychological Science, 16(12), 932-936.

https://doi.org/10.1111/j.1467-9280.2005.01639.x

Smith-Keiling, B. L., \& Hyun, H. I. F. (2019). Applying a computer-assisted tool for semantic analysis of writing: Uses for STEM and ELL. Journal of Microbiology \& Biology Education, 20(1).

https://doi.org/10.1128/imbe.v20i1.1709

Sonnenschein, A. R., Hofmann, S. G., Ziegelmayer, T., \& Lutz, W. (2018). Linguistic analysis of patients with mood and anxiety disorders during cognitive behavioral therapy. Cognitive Behaviour Therapy, 47(4), 315-327. https://doi.org/10.1080/16506073.2017.1419505

Sullivan, F. R., Kapur, M., Madden, S., \& Shipe, S. (2015). Exploring the role of 'gendered' discourse styles in online science discussions. International Journal of Science Education, 37(3), 484-504.

https://doi.org/10.1080/09500693.2014.994113

Tadajewski, M., \& Hewer, P. (2011). Intellectual contributions and gap-spotting. Journal of Marketing Management, 27(5-6), 449-457. https://doi.org/10.1080/0267257X.2011.562364

Tausczik, Y. R., \& Pennebaker, J. W. (2010). The psychological meaning of words: LIWC and computerized text analysis methods. Journal of Language and Social Psychology, 29(1), 24-54.

https://doi.org/10.1177\%2F0261927X09351676

Vaughn, L. A. (2018). Contents of hopes and duties: A linguistic analysis. Frontiers in Psychology, 9, Article 757. https://doi.org/10.3389/fpsyg.2018.00757

Waters, T. E., Steele, R. D., Roisman, G. I., Haydon, K. C., \& Booth-LaForce, C. (2016). A linguistic inquiry and word count analysis of the Adult Attachment Interview in two large corpora. Canadian Journal of Behavioural Science/Revue Canadienne des Sciences du Comportement, 48(1), 78-88.

https://dx.doi.org/10.1037\%2Fcbs0000035

Wilson, A. (2013). Embracing Bayes factors for key item analysis in corpus linguistics. In M. Bieswanger \& A. Koll-Stobbe (Eds.), New approaches to the study of linguistic variability (pp. 3-11). Peter Lang. https://eprints.lancs.ac.uk/id/eprint/51045

Wolohan, J. T., Hiraga, M., Mukherjee, A., Sayyed, Z. A., \& Millard, M. (2018, August). Detecting linguistic traces of depression in topic-restricted text: Attending to self-stigmatized depression with NLP. In Proceedings of the First International Workshop on Language Cognition and Computational Models (pp. 11-21). Association for Computational Linguistics. https://www.aclweb.org/anthology/W18-4102.pdf 
Zilcha-Mano, S., Muran, J. C., Hungr, C., Eubanks, C. F., Safran, J. D., \& Winston, A. (2016). The relationship between alliance and outcome: Analysis of a two-person perspective on alliance and session outcome. Journal of Consulting and Clinical Psychology, 84(6), 484-496.

https://doi.org/10.1037/ccp0000058 


\section{Table 1.1}

Rupture Type Descriptive Statistics (RQ1) and Results from Inferential Analyses (RQ2)

\begin{tabular}{|c|c|c|c|c|c|c|c|c|c|c|}
\hline \multirow[b]{2}{*}{ Var. } & \multirow[b]{2}{*}{ Cat. } & \multicolumn{3}{|c|}{ Actual Count } & \multicolumn{3}{|c|}{ Expected Count } & \multirow[b]{2}{*}{$G^{2}$} & \multirow[b]{2}{*}{$\mathrm{BIC}$} & \multirow[b]{2}{*}{ BIC Desc. } \\
\hline & & Confr. & Withd. & Mix. & Confr. & Withd. & Mix. & & & \\
\hline shehe & Ling. & 2.98 & 40.00 & 28.85 & 19.05 & 22.70 & 30.08 & 31.85 & 14.01 & Very Strong \\
\hline certain & Psy. & 46.08 & 18.94 & 33.87 & 26.22 & 31.25 & 41.41 & 19.36 & 1.52 & Weak \\
\hline negemo & Psy. & 31.97 & 48.05 & 101.92 & 48.25 & 57.51 & 76.19 & 15.74 & -2.11 & Positive \\
\hline i & Ling. & 177.95 & 306.05 & 348.10 & 220.66 & 262.99 & 348.44 & 15.56 & -2.29 & Positive \\
\hline $\operatorname{anx}$ & Psy. & 6.95 & 13.02 & 36.06 & 14.86 & 17.71 & 23.46 & 12.43 & -5.41 & Positive \\
\hline differ & Psy. & 93.94 & 89.00 & 169.97 & 93.59 & 111.54 & 147.78 & 8.08 & -9.77 & Strong \\
\hline discrep & Psy. & 60.97 & 47.10 & 64.92 & 45.87 & 54.68 & 72.44 & 6.41 & -11.43 & Very Strong \\
\hline sad & Psy. & 5.96 & 4.02 & 16.93 & 7.14 & 8.51 & 11.27 & 5.61 & -12.23 & Very Strong \\
\hline cause & Psy. & 38.93 & 27.93 & 47.98 & 30.45 & 36.30 & 48.09 & 4.26 & -13.58 & Very Strong \\
\hline they & Ling. & 4.97 & 0.95 & 4.08 & 2.65 & 3.16 & 4.18 & 3.75 & -14.09 & Very Strong \\
\hline tentat & Psy. & 114.00 & 108.88 & 148.96 & 98.61 & 117.52 & 155.71 & 3.23 & -14.61 & Very Strong \\
\hline posemo & Psy. & 38.93 & 61.07 & 84.04 & 48.81 & 58.17 & 77.07 & 2.91 & -14.94 & Very Strong \\
\hline anger & Psy. & 11.92 & 22.96 & 28.85 & 16.90 & 20.14 & 26.69 & 2.19 & -15.65 & Very Strong \\
\hline we & Ling. & 6.95 & 9.94 & 15.99 & 8.72 & 10.39 & 13.77 & 0.75 & -17.10 & Very Strong \\
\hline
\end{tabular}

Note. Confrontation $n=1,986$, withdrawal $n=2,367$; mixed $n=3,136$; adjusted error rate for 14 comparisons was $p<.00007 ; G^{2}$ for that error rate $=19.09$. A negative $\mathrm{BIC}$ indicates support for the null hypothesis. 
Table 1.2

Post Hoc Pairwise Rupture Comparisons

\begin{tabular}{lcccccccc}
\hline & \multicolumn{9}{c}{ Corpus 1} & \multicolumn{2}{c}{ Corpus 2} \\
Category & Process & Type & Raw Ct. & Type & Raw Ct.. & $G^{2}$ & BIC & BIC Descript. \\
\hline shehe & Ling. & W & 40 & C & 2.98 & 31.77 & 23.39 & Very Strong \\
certain & Psych. & W & 18.94 & C & 46.08 & 16.95 & 8.57 & Strong \\
shehe & Ling. & M & 28.85 & C & 2.98 & 14.17 & 5.63 & Positive \\
certain & Psych. & M & 33.87 & C & 46.08 & 11.58 & 3.04 & Positive \\
\hline
\end{tabular}

Note. The critical value for $G^{2}$ at $p<.001$ is 10.83 . Withdrawal $n=2,367$; confrontation $n=$ 1,986; mixed $n=3,136$. 


\section{Table 1.3}

Rupture Versus Reference Corpus Results (RQ3)

\begin{tabular}{llccccc}
\hline Category & Process & \multicolumn{2}{c}{ Corpus } & $\mathbf{G}^{\mathbf{2}}$ & BIC & $\begin{array}{c}\text { BIC } \\
\text { Descriptors }\end{array}$ \\
& & $\begin{array}{c}\text { Rupture } \\
\text { Raw Ct } \\
\text { (Norm count) }\end{array}$ & $\begin{array}{c}\text { Reference } \\
\text { Raw Ct } \\
\text { (Norm count) }\end{array}$ & & & \\
\hline posemo & Psych & $184.04(2.46)$ & $594.23(4.38)$ & 51.49 & $41.54^{*}$ & Very Strong \\
discrep & Psych & $172.99(2.31)$ & $497.91(3.67)$ & 29.47 & $19.52^{*}$ & Very Strong \\
tentat & Psych & $371.84(4.97)$ & $518.26(3.82)$ & 14.63 & 4.68 & Positive \\
they & Ling & $9.99(0.13)$ & $55.62(.41)$ & 13.57 & 3.62 & Positive \\
\hline
\end{tabular}

Note. The critical value for $G^{2}$ at $p<.001$ is 10.83. Rupture $n=7,489$; reference $n=13,567$. 